\title{
Comparison of data processing techniques for single-grating $x$-ray Talbot interferometer data
}

Shashidhara Marathe, Marie-Christine Zdora, Irene Zanette, Silvia Cipiccia, Christoph Rau

Shashidhara Marathe, Marie-Christine Zdora, Irene Zanette, Silvia Cipiccia, Christoph Rau, "Comparison of data processing techniques for single-grating X-ray Talbot interferometer data," Proc. SPIE 10391, Developments in X-Ray Tomography XI, 103910S (11 October 2017); doi: 10.1117/12.2274021

SPIE Event: SPIE Optical Engineering + Applications, 2017, San Diego, California, United States 


\title{
Comparison of Data Processing Techniques for Single Grating X-ray Talbot Interferometer Data
}

\author{
Shashidhara Marathe ${ }^{\mathrm{a}}$, Marie-Christine Zdora ${ }^{\mathrm{a}, \mathrm{b}}$, Irene Zanette ${ }^{\mathrm{a}}$, Silvia Cipiccia ${ }^{\mathrm{a}}$ and Christoph Rau ${ }^{\mathrm{a}}$ \\ ${ }^{a}$ Diamond Light Source, Harwell Science and Innovation Campus, Didcot, Oxfordshire \\ OX11 0DE, United Kingdom \\ ${ }^{\mathrm{b}}$ Department of Physics \& Astronomy, University College London, London WC1E 6BT, United \\ Kingdom
}

\begin{abstract}
The phase-stepping (PS) mode of the X-ray Grating Talbot interferometer (XGTI) data processing technique ${ }^{1,2}$ can yield high-spatial resolution images, albeit with lower throughput since each projection of a tomogram requires at least five phase-stepping images. To overcome this problem, we can use a setup with only a single phase grating in combination with a high-resolution detector system and Spatial Harmonic Imaging (SHI) technique ${ }^{3}$. The latter technique makes use of the fact that a Talbot interferogram consists of carrier frequency spectrum of the grating overlapping with the sample spectrum and by Fourier transforming the interferogram we can separate the two ${ }^{3}$. The disadvantage of this is that the spatial resolution is inferior. In this manuscript we will compare these two single grating data processing techniques using a single data set of a mouse embryo heart and discuss advantages and disadvantages of each technique. These two techniques can be used as complementary; one for high resolution, and the other for high-speed imaging.
\end{abstract}

Keywords: $x$-ray grating interferometer, phase-contrast imaging, phase-stepping technique, Spatial Harmonic Imaging

\section{INTRODUCTION}

For over a decade, XGTI has been at the forefront of X-ray phase-contrast imaging due to its ability to visualize very small density variations of bulk objects with the possibility of extracting three imaging modalities from a single interferogram image or a data set. Most widely used XGTI data acquisition techniques, namely the two grating phasestepping and moiré techniques, suffer from poor signal-to-noise ratio (SNR), which is mainly due to about $50 \%$ reduction in scattered sample signal by the second amplitude grating. Consequently, longer exposure times are required for an improved SNR, which comes at the cost of an increased dose to the sample.

A single grating phase-stepping measurement of a mouse embryo with excellent contrast was previously measured by M-C. Zdora et $\mathrm{al}^{4}$, at Diamond Light Source Ltd., I13-2 beamline. Here we discuss two data processing techniques that can be used with a single-grating setup, namely the PS technique and SHI technique. The PS technique has been used for high-contrast phase imaging for about a decade ${ }^{1-2,5-6}$. The SHI technique, which was introduced to the $\mathrm{x}$-ray phasecontrast imaging field by H.Wen ${ }^{3}$, has been used for x-ray beam coherence measurements, as a sensor element and for imaging of burning of a fire retardant material ${ }^{7-10}$. Both techniques have pros and cons. We will evaluate the four major properties - spatial resolution, fast imaging, reconstruction of the probe and differential phase contrast and phase wrapping. Section 2 will briefly elucidate the theoretical background and point to the references for comprehensive reading. This will be followed by the experimental details (Section 3), results (Section 4) and the conclusions.

\section{THEORETICAL BACKGROUND}

The mathematical formalism for both the techniques, namely the PS and the SHI techniques that we used in this manuscript, is provided below.

Suppose a phase grating is placed in the beam, the replicated images of the grating pattern are periodically reproduced at distances, called self-imaging distances $\left(d_{f t}\right)$, after the grating due to the Talbot effect ${ }^{11}$. For a $1 \mathrm{D} \pi / 2$ checkerboard phase grating, the fractional Talbot distances are given by

Developments in X-Ray Tomography XI, edited by Bert Müller, Ge Wang, Proc. of SPIE Vol. 10391,

103910S - (C) The Authors. Published under a Creative Commons Attribution CC-BY 3.0 License · doi: 10.1117/12.2274021 


$$
d_{f t}=\frac{n p^{2}}{2 \eta^{2} \lambda}
$$

where $n=1,3,5 \ldots, \eta=1$ for $\pi / 2$ phase shift grating, $p$ is the period of the grating, and $\lambda$ is the $\mathrm{x}$-ray wavelength. Typically, the interference pattern period is of a few microns in size.

\subsection{Phase-stepping Technique}

Stepping a phase grating using a step size of fractions of period of a grating we can create an intensity profile for each step of the phase grating, which can be written in mathematical terms as,

$$
I=a_{0}+a_{1} \cos \left(\frac{2 \pi x_{g}}{p_{2}}+\phi\right)
$$

Here, $I$ is the intensity pattern of a single pixel, $a_{0}$ and $a_{1}$ are the first and second coefficients of the Fourier transform or fitting function. $x_{g}$ is the phase stepping period, $p_{2}$ the period of the interferogram at the detector position, and $\phi$ the phase of the sinusoidal curve representing the phase stepping. Measuring with sample and without sample in the beam we can obtain two phase stepping curves and deduce the Absorption $(A)$, differential phase contrast $(D P C)$ and darkfield $(D F)$ images by comparing the two curves, as given below,

$$
A=1-\frac{a_{0}^{s}}{a_{0}^{r}}, D P C=\phi^{s}-\phi^{r}, D F=\frac{a_{0}^{r} a_{1}^{s}}{a_{0}^{s} a_{1}^{r}} .
$$

Here, superscripts, ' $s$ ' and ' $r$ ' represent images taken with and without the sample in the beam, respectively. Evaluating this for all of the pixels we can build three image contrast modes representing different physical characteristics of the sample. For a thorough reading please check $^{1,2}$ and the references there in.

\subsection{Harmonic imaging technique}

If we place a sample upstream of a phase grating, the interferogram at the fractional Talbot distance is proportional to the product of the transmittance of the object with the grating pattern ${ }^{3,9}$, which is given by,

$$
w_{g}(x, y)=w(x, y) \times g(x, y) .
$$

Here we have assigned $w(x, y)$ and $g(x, y)$ as the transmittance functions of the sample and the phase grating, respectively. Therefore, the Fourier transform $\left(W_{g}(k)\right)$ of the measured interferogram is convolution of the spectrum of the sample with the spectrum of the self-image of the grating pattern. For a 1D grating with its grid lines aligned in $y$ direction, parallel to lab vertical, we have

$$
W_{g}(k)=W(k) * G(k)=\sum_{m} G_{m} W\left(k_{y}, k_{x}-\frac{2 \pi m}{p}\right) .
$$

Here, $W_{g}(k), F(k)$ and $G(k)$ represent the Fourier transform of $w_{g}(x, y), w(x, y)$ and $g(x, y)$, respectively. $G_{m}$ is the amplitude of the $m^{\text {th }}$ order harmonic peak. The pattern around the harmonic peaks represents the spectrum of the entire sample, assuming that the harmonic spectra from adjacent peaks do not overlap, or the high-frequency components of the sample are negligible. We can then obtain the $m^{\text {th }}$ order spatial harmonic image $h_{m}(x, y)$ by the inverse Fourier transform of the $m^{\text {th }}$ order spectrum peak within the interval $k_{x} \in[\pi(2 m-1) / p, \pi(2 m+1) / p]$, which leads to

$$
h_{m}(x, y)=G_{m} w(x, y)
$$

It can be inferred from Eq. 6 that the harmonic images are identical containing the information of the entire sample and are only weighted by the amplitude of the harmonic peak of the grating grid. The phase of the high-order $(\mathrm{m}>0)$ harmonic images is influenced by the x-ray refraction of the sample and one can extract differential phase images of the sample by inverse Fourier transforming the harmonic spectrum. The details can be found in Ref. 3 and references there in. 


\section{EXPERIMENT}

The experiment was performed with $15 \mathrm{keV}$ X-ray energy produced from a combination of 'Si' mirror and a MultiLayer Monochromator (MLM)-Ruthenium stripe, at the I13-Imaging branch beamline of Diamond Light Source Ltd, UK. The MLM is under commissioning and being used for the first time for a grating interferometer experiment here at the I13-imaging branch. We used a 1D phase grating of $4.785 \mu \mathrm{m}$ nominal period, which gives a $3 \pi / 2$ phase shift at 15 $\mathrm{keV}$ X-ray energy. The detector was placed at $41.6 \mathrm{~cm}$ from phase grating, corresponding to $3^{\text {rd }}$ Talbot distance. A Pco.edge5.5 camera was used with a $4 \mathrm{x}$ objective. Accounting for the magnification created by the objective and the optical magnification of the detector system the single effective pixel size of the detector was $0.81 \mu \mathrm{m} \times 0.81 \mu \mathrm{m}$. Images were collected with exposure times of $0.1 \mathrm{~s}, 2000$ projections over $180^{\circ}$ at an interval of $0.09^{\circ}$.

The phase-stepping scan was performed with 5 steps per projection over one period of the interference pattern and taking dark and flats for every 100 projections. This is done to account for the drift and vibration issue of the MLM. For the SHI technique we used the $1^{\text {st }}$ phase stepping image of each projection for the analysis.

\section{RESULTS}

The main goal of this paper was to compare the processing techniques by using the same data set and come up with an understanding of the strong points of each of the techniques. The parameters that we looked at are spatial resolution, fast imaging, reconstruction of the probe and differential phase contrast \& phase wrapping. Below we have elaborated on each of the parameters that we used for evaluating the techniques.

\subsection{Spatial Resolution}

In the present experiment a 1D phase grating with lines in vertical direction was used. Therefore, the peaks in the Fourier-transformed image can be found in the horizontal direction. The distance between the $0^{\text {th }}$ and $1^{\text {st }}$ harmonic peak is 429 pixels. As described in Section 2.2, the maximum distance from a peak in the horizontal direction without overlapping of the harmonic spectra is half the distance between two peaks. Hence we selected harmonic images with a width of 427 pixels symmetrically around the center of the peaks. Even though the height of the harmonic images can be as large as the original image itself, here we choose it similar to the width. As can be recognized, for the PS technique, the whole height and width of the original image (i.e., $2560 \times 2160$ pixels) is used for the image formation. Therefore the resolution of the SHI technique is about $6 \times 5$ times inferior to the PS technique in the width and height direction, respectively.
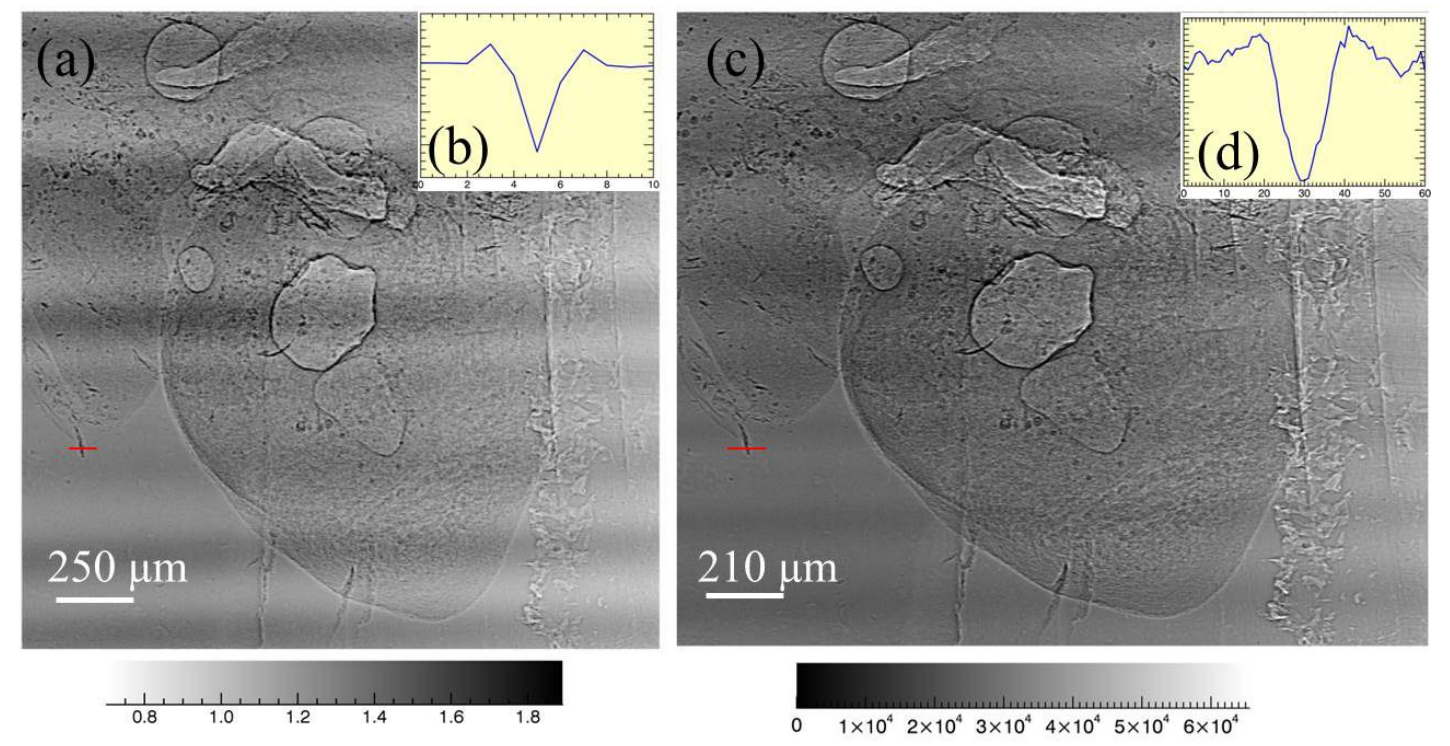

Figure 1. The absorption image of mouse embryo heart reconstructed from SHI (a) and PS (c) techniques. The colour bar represents arbitrary units. The inset (b) and (d) are the line profiles across red horizontal line in (a) and (b), respectively, comparing the spatial resolution. 
Figure 1 shows the absorption image of the mouse embryo heart sample processed with SHI (see Fig. 1(a)) and the PS (see Fig. 1(c)) techniques. The insets Fig. 1(b) and Fig. 1(d) compare the spatial resolution of the image across the red lines in Fig. 1(a) and Fig. 1(c). The line profile across the feature is represented by 4 and 24 pixels in Fig. 1(b) and Fig. $1(d)$, respectively, indicating a 6 times inferior spatial resolution in horizontal (width) direction.

\subsection{Fast Imaging}

As described in Section 2 the PS technique uses 5 images per projection to reconstruct a single projection of the tomogram. However, the SHI technique uses only one image per projection. Considering the tomography data acquisition this implies the SHI technique is also suitable for fly-scans where the sample rotation stage is continuously rotated in synchronization with camera acquisition, reducing the dead time for rotation stage movements after every projection. The exposures needed for SHI is almost same as normal absorption imaging since the phase grating absorption is low. Using a fly-scan mode and $0.5 \mathrm{~s}$ exposure time, the SHI technique is typically 8 times quicker than the PS. When comparing PS and SHI, the PS requires an exposure time of $0.1 \mathrm{~s} /$ image (total $0.5 \mathrm{~s} /$ projection), while 0.5 $\mathrm{s}$ /image for the SHI. This way we have same exposure per projection. However, it is important to note that the images from the SHI technique shown here were reconstructed from single image of $0.1 \mathrm{~s}$ exposure time (i.e., the $1^{\text {st }}$ images from a phase-stepping set of images for each projection). This implies we can further improve the signal-to-noise ratio for SHI.

Also we can use the SHI technique to do a quick scan of the sample if the sample structure is unknown or if we are looking at a particular region within a sample where we want to do high spatial resolution imaging using PS. This may be important for dose-sensitive samples.

\subsection{Reconstruction of probe with SHI}

One of the unique features of the SHI technique is that we can obtain the phase profile of the illuminating beam. What we mean by this is that when we take the reference images without the sample in the beam, we can process this in a similar way as we do to the sample images to obtain the amplitude profile of $0^{\text {th }}$ and $1^{\text {st }}$ harmonic image as well as the differential phase contrast (DPC) image of the $1^{\text {st }}$ harmonic image. For the PS technique, to obtain a phase image, you need both the reference as well as the sample image. One can argue that the reference image from the PS measurement can also be used to process like the SHI technique to obtain the phase images, but what we are saying is that it is not integral to the PS system. However, for the SHI technique it is one of the processes in the chain.
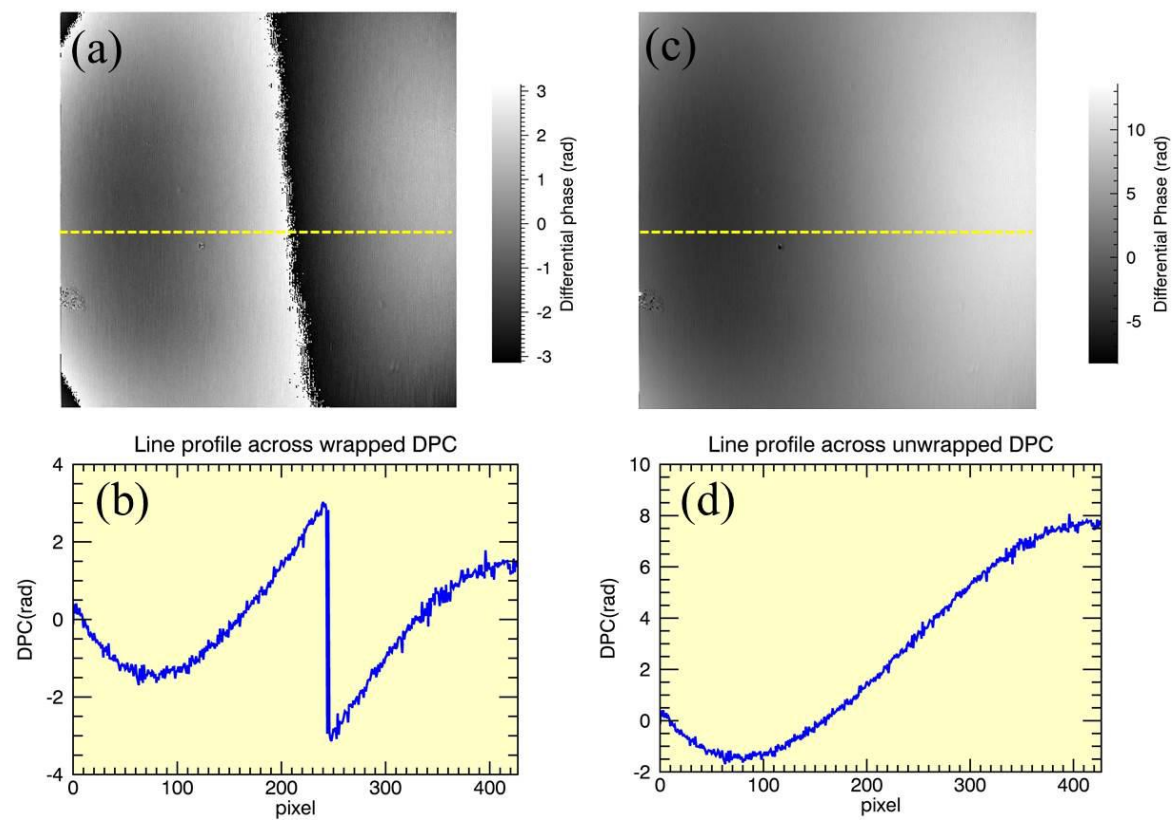

Figure 2. The DPC of the reference image obtained using SHI technique. (a) Wrapped DPC ( $427 \times 427$ pixels), (b) line profile across (a) at $y=200^{\text {th }}$ pixel. (c) Unwrapped DPC, (d) line profile across (c) at $y=200^{\text {th }}$ pixel. 
Getting the phase information of the illuminating beam can be very useful for reconstruction of the wavefront. It may also be useful to correct for the wavefront aberrations by having an adaptive optics element in a feed-back loop with the single-grating harmonic imaging interferometer [9]. The known wavefront information from the reconstruction can also be fed to the algorithms for phase reconstruction. Figure 2 illustrates the DPC of the reference image obtained using SHI technique. Fig. 2(a) shows the wrapped DPC image which has a size of $427 \times 427$ pixels and Fig. 2(b) the line profile across (a) at $y=200^{\text {th }}$ pixel. Fig. 2(c) displays the unwrapped DPC and Fig. 2(d) the line profile across (c) at $y=200^{\text {th }}$ pixel.

\subsection{Differential phase contrast and phase wrapping}

The pco.edge 5.5 camera has a chip size of 2560 x 2160 pixels. The SHI technique makes use of only $427 \times 427$ pixels to plot the same image. In the case of the SHI technique it has an effect of binning since the information spread over $6 \times 5$ pixels in the original image is merged into single pixel in SHI. Figures 3(a) and 3(c) and Figs. 3(b) and 3(d) show the DPC images obtained from the SHI and PS techniques, respectively, for two different orientations of the sample. Figures 3 (a) and 3(c) were produced by phase unwrapping both the reference and the sample DPC images. Figures 3(a) and 3(c) have similar contrast compared to Fig. 3(b) and Fig. 3(d), however the spatial resolution is better for the latter. In Fig. 3(b) and 3(d) the white arrow indicates the position of a horizontal band of very faint vertical lines, which is due to some instabilities in the setup. The beam from the MLM has a horizontal stripe structure, typical for this type of monochromators. A small drift of the beam during the measurement caused this horizontal band.
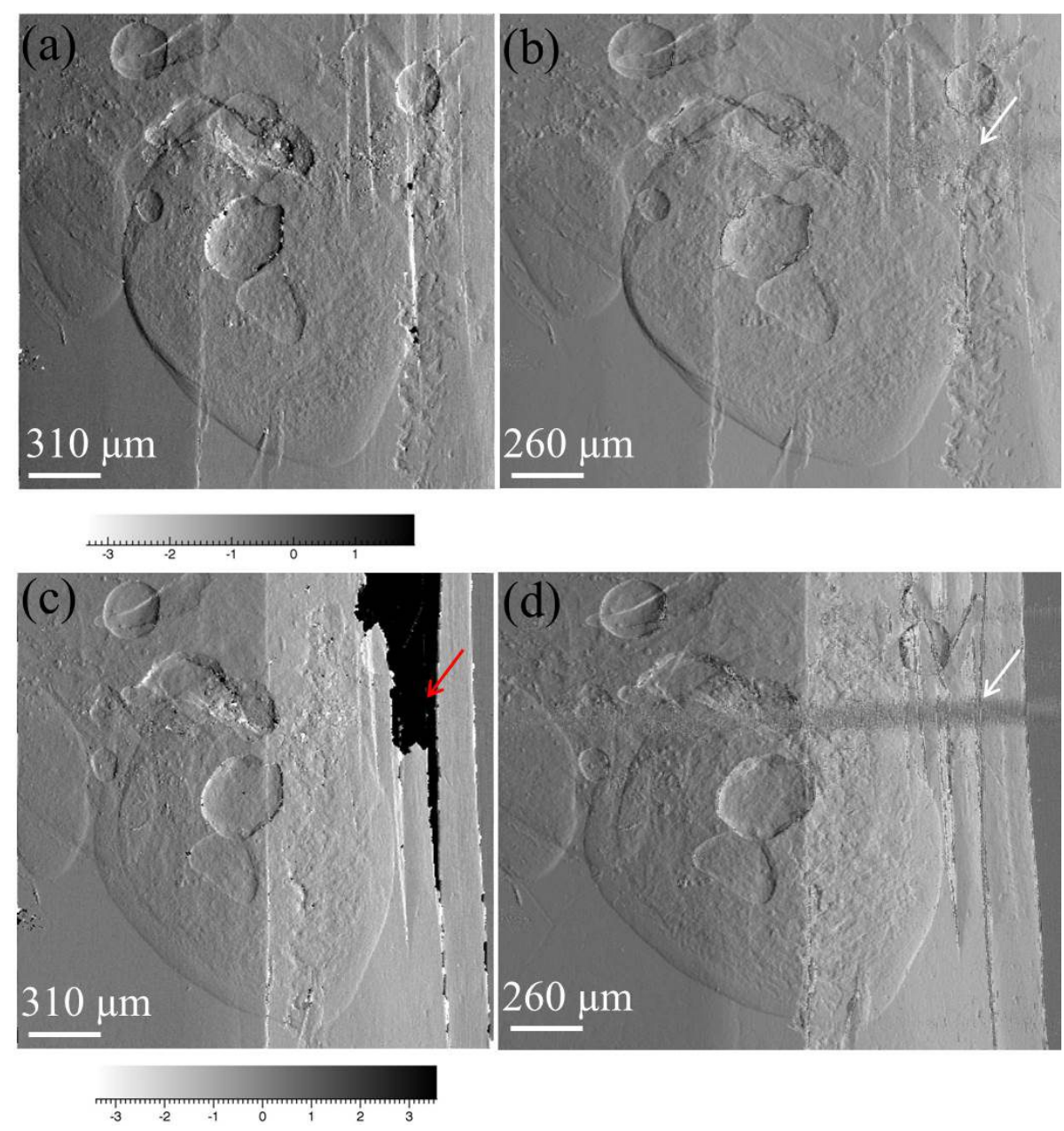

Figure 3. DPC images of a mouse embryo heart reconstructed with the SHI $(a, c)$ and the PS (b,d) techniques at $0^{\circ}(\mathrm{a}, \mathrm{b})$ and $12.42^{\circ}(\mathrm{c}, \mathrm{d})$ rotation of the sample. The effect of phase wrapping is visible in (c), indicated by the red arrow. A horizontal band of vertical lines in (b) and (d) is caused by beam drift. The colour bar is in radians. 
In the case of SHI technique we are measuring the differential phase image (DPC) of the reference image and subtracting this image from the sample DPC to produce the final DPC image. The change in DPC image with beam drift is small; hence the SHI technique is more robust to beam instabilities. Also the inferior spatial resolution of the SHI technique helps to reduce this effect. However, for the PS technique we compare the intensity profiles of reference and sample images which are much more prone to this beam drift.

The black region (indicated by the red arrow) in the top right hand side of the DPC image of Fig. 3(c) is the incomplete phase-unwrapping of the image. Figure 3(d) shows images processed with the PS technique at the same orientation of the sample. The phase-wrapping issue seems less pronounced than for the SHI technique. We attribute this to the lower spatial resolution of the SHI technique; however more quantitative analysis is needed.

\section{CONCLUSIONS}

Here we have compared the PS and SHI processing techniques for grating interferometry with a single grating to understand their relative merits and demerits. It can be seen that the spatial resolution for the PS technique is limited by the detector pixel size, the detector point spread function as well as the grating period. Whereas for the SHI technique the spatial resolution is inferior at least 4-5 times in comparison with PS. One of the advantages of the SHI technique is that it can operate at lower dose and is useful for fast imaging. For the setup mentioned here it is estimated to be 8 times faster with further 5 times reduction of total exposure time possible, depending on whether we use a single image for faster imaging or several images for better statistics. It is shown that the reconstruction of the probe is possible with the SHI technique and it may be useful for data analysis, or to tweak the incident beam using an adaptive optics feedback loop. DPC images from SHI have good contrast, but can suffer from phase-wrapping issues. Further studies with a reference sample and a more stable beam are planned.

\section{ACKNOWLEDGEMENTS}

Authors gratefully acknowledge the use of Diamond Light Source on the beamline I13, Diamond-Manchester Branchline. We would like to acknowledge Dr. Andrew C. Cook, Daniel Dilg, UCL Institute of Cardiovascular Science, UK for allowing us to use their sample for testing. We would also like to thank Dr. Kaz Wanelik, Andy Wilson and Simon Logan, Diamond Light Source, UK, for their help during the experiment and data analysis.

\section{REFERENCES}

[1] Weitkamp, T., Diaz, A., David, C., Pfeiffer, F., Stampanoni, M., Cloetens, P., Ziegler, E., "X-ray phase imaging with a grating interferometer," Opt. Express 13, 6296-6304 (2005).

[2] Momose, A., Kawamoto, S., Koyama, I., Hamaishi, Y., Takai, K., Suzuki, Y., "Demonstration of X-Ray Talbot Interferometry," Jpn. J. Appl. Phys. 42, L866-L868 (2003).

[3] Wen, H. H., Bennett, E. E., Kopace, R., Stein, A. F., Pai, V., "Single-shot x-ray differential phase-contrastand diffraction imaging using two-dimensional transmission gratings," Opt. Lett., 35, 1932-1934 (2010).

[4] Zdora, M-C., Vila-Comamala, J., Schulz, G., Khimchenko, A., Hipp, A., Cook, A. C., Dilg, D., David, C., Grünzweig, C., Rau, C., Thibault, P., Zanette, I., "X-ray phase microtomography with a single grating for highthroughput investigations of biological Tissue," Biomed. Opt. Express, 8, 1257-1270 (2017).

[5] Pfeiffer, F., Bech, M., Bunk, O., Kraft, P., Eikenberry, E. F., Brönnimann, C., Grünzweig, C., David, C., "Hard-Xray dark-field imaging using a grating interferometer," Nat. Mater. 7(2), 134-137 (2008)

[6] Zanette, I., Weitkamp, T., Donath, T., Rutishauser, S., David, C., "Two-dimensional X-ray grating interferometer," Phys. Rev. Lett. 105(24), 248102 (2010).

[7] Marathe, S., Shi, X., Wojcik, M. J., Kujala, N. G., Wen, H., Divan, R., Mancini, D. C., Macrander, A. T., and Assoufid, L., "Probing transverse coherence of x-ray beam with 2-D phase grating interferometer," Opt. Express 22, 14041 (2014).

[8] Shi, X., Marathe, S., Wojcik, M. J., Kujala, N. G., Macrander, A. T., Assoufid, L., "Circular grating interferometer for mapping transverse coherence area of X-ray beams," Appl. Phys. Lett. 105, 041116 (2014).

[9] Marathe, S., Shi, X., Khounsary, A. M., Wojcik, M. J., Kujala, N. G., Macrander, A. T., Assoufid, L., "Development of single grating X-ray Talbot interferometer as a feedback loop sensor element of an adaptive X-ray mirror system," Proc. SPIE 9208, Adaptive X-Ray Optics III, 92080D (2014) 
[10] Olatinwo, M. B., Ham, K., McCarney, J., Marathe, S., Ge, J., Knapp, G., Butler, L.G., "Analysis of Flame Retardancy in Polymer Blends by Synchrotron X-ray K-edge Tomography and Interferometric Phase Contrast Movies," J. Phys. Chem. B , 120, 2612-2624 (2016).

[11] Talbot, H. F., "LXXVI. Facts relating to optical science. No. IV," Philos. Mag. 9, 401-407 (1836). 